\title{
Stay on the Ball! An Interaction Pattern Approach to the Engineering of Motivation
}

\author{
Kirstin Kohler $^{1}$, Sabine Niebuhr ${ }^{1}$, and Marc Hassenzahl ${ }^{2}$ \\ ${ }^{1}$ Fraunhofer IESE, Fraunhofer-Platz 1, 67663 Kaiserslautern, Germany \\ \{kohler, niebuhr\}@iese. fraunhofer.de \\ ${ }^{2}$ University of Koblenz-Landau, Fortstr. 7, 76829 Landau, Germany \\ hassenzahl@uni-landau.de
}

\begin{abstract}
This paper introduces an interaction pattern approach to "engineer" motivation. Its goal is to provide concrete ideas (in the form of patterns) on how to design software that motivates its users to stay on a task. The paper presents an example of a motivating pattern, the Task Status Display (TSD), and its empirical validation. Preliminary results support its motivational effect, thereby lending support to the general notion of building motivation into interactive products for the workplace.
\end{abstract}

\section{Introduction}

Traditional usability engineering (UE) approaches (e.g., [1]) take a predominantly task-oriented perspective on designing interactive systems. They assume given behavioral goals (do-goals, e.g., "make a telephone call") and attempt to design systems in a way to optimally support goal accomplishment. However, we argue that this approach misses out on at least one important aspect of human behavior namely motivation.

There is an implicit assumption of UE that in the work context, goals (i.e., tasks) are provided externally and are relatively fixed. And indeed, a production line worker does not have much choice other than to perform the given task or to quit her job. However, there are other work domains where situations are less clear cut. In many work contexts, such as office work, individuals will be given relatively abstract goals; how to actually pursue them is often their choice. In addition, people often have more than one pending task, which allows for subtle shifts in priorities. Thus, whenever individuals have the autonomy to procrastinate or even fully abandon boring tasks, motivation will become extremely important.

Game design and e-learning design can be interesting sources of motivational principles in design. Both spend much effort on making their products as appealing as possible, predominantly because people will use them on a voluntary basis. Especially games are very good at motivating repetitive behavior, which would be considered as boring in a work context.

Nevertheless, it is a long way from broad principles to concrete design solutions. To bridge this gap, we use interaction patterns. Patterns describe approved solutions to recurring design problems in a structured way. By that, they store and provide 
design knowledge. The use of pattern has become increasingly common since their transfer from architecture to software and usability engineering (e.g.[2,3]). However, currently there are no patterns describing solutions to motivational or other hedonic aspects.

\section{An Example: The Task Status Display}

Tidwell [4] suggested a pattern called "Status Display". Her intention was to keep the user informed about what happens during interaction. We drew upon this general idea to develop the "Task Status Display" (TSD). TSD visualizes the current goal, shows the extent to which it has already been accomplished, which further steps have to be taken and how big these steps are (for more details see [5]). TSD visualizes the goal and goal progress. Its

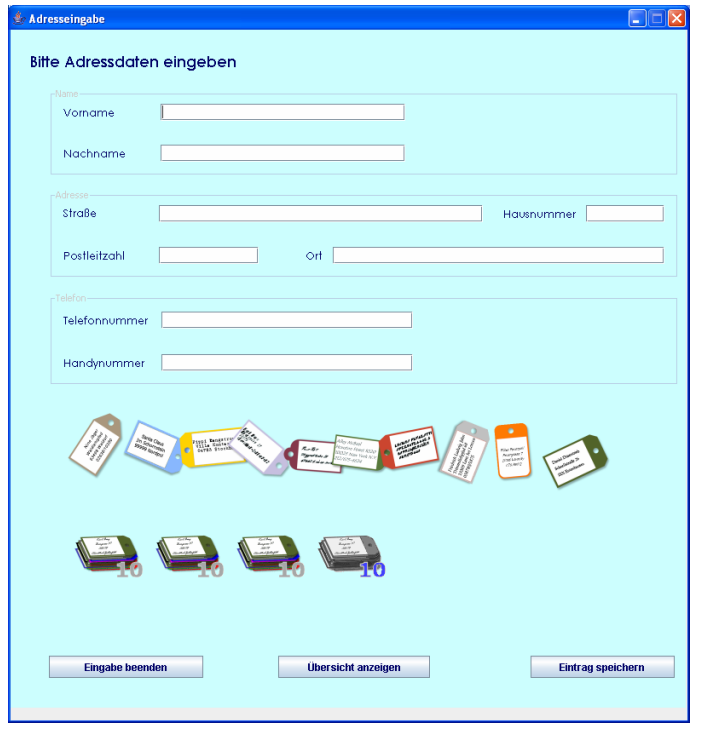

Fig. 1. Address database interface with TSD motivating effect is assumed to emerge from (1) helping users to stay focused on their goal, or even by providing an alternative, more interesting "substitute" goal, and from (2) providing feedback to adjust performance [6]. Note that although superficially similar, TSD is not comparable to a progress bar. A progress bar rather provides feedback about the system's state ("Don't worry, I'm working") than the user's state in the sense of goal accomplishment.

A TSD can be implemented in different ways [5]. In order to explore the potential motivating effect, we set up a simple address database with TSD as a decreasing amount of address cards (see fig 1). The system's main purpose is to enter a fixed number of addresses into a database. With every address added, a card disappears from the row; for every ten addresses, one card stack disappears and the row is filled with new cards.

\section{Does It Work?}

We prepared two versions of the address entry mask; one version with TSD, the other without. Fourteen individuals ( 4 female, median age $=26, \min =20, \max =35$ ) were randomly assigned to either the version with TSD ("with pattern", N=6) or without ("without pattern", $\mathrm{N}=7$ ). All participants were asked to enter 40 addresses provided as scanned business cards. They were further told that they had a limit of 75 minutes 
to accomplish the task. The session was either ended by reaching the limit of 75 minutes or by having entered all addresses. In addition, they were encouraged to take breaks whenever they felt like doing so. To let the break appear a little more tempting, the desktop gave easy access to a number of games (e.g., Pacman, Sudoku) and current news (both provided by Google).

We were interested in two aspects, namely the impact of the pattern on system perceptions and evaluations (i.e., subjective) and on task persistence (i.e., objective). System perceptions and evaluations were measured with the AttrakDiff2Questionnaire [7]. It distinguishes between perceived pragmatic quality (PQ), hedonic quality, and appeal (APPEAL). PQ is the perceived ability of a product to support the efficient and successful accomplishment of "do-goals", such as to "enter an address". In contrast, hedonic quality is the perceived ability of a product to support so-called "be-goals", such as "be something special", "be cool". Specifically, we focused on hedonic quality stimulation (HQS), a measure of the system's perceived novelty, uniqueness, and excitation. APPEAL is the general evaluation of the system as either good or bad. In general, each aspect was measured by seven single bipolar items, averaged to a single scale value (see Tab. 1 for measures of internal consistency). Example items are "confusing-structured" (PQ), "dull-captivating" (HQS), and "badgood" (APPEAL). The participants were asked to fill in the questionnaire at the end of the session. Task persistence was operationalized simply by the number of breaks taken during address entry. A break was defined as switching from the address entry mask to one of the offered games or news.

In general, we expected participants to perceive the employed pattern as primarily stimulating (HQS) and not so much as adding to its pragmatic quality (i.e., usability). Nevertheless, an effect of the pattern on task persistence should become apparent, with less breaks in the "with pattern"-condition. Specifically, stimulation was expected to be linked directly (and inversely) to task persistence.

Table 1 shows the means and standard deviation of all measures for the "with pattern" and "without pattern" conditions.

Table 1. Means (standard deviation) for all measures, separately for the with-pattern and without-pattern conditions

\begin{tabular}{|c|c|c|c|}
\hline Measure & $\begin{array}{c}\text { Condition/System } \\
\text { with pattern }(\mathrm{N}=6)\end{array}$ & without pattern $(\mathrm{N}=7)$ & $\begin{array}{l}\text { Mann- } \\
\text { Whitney }\end{array}$ \\
\hline \multicolumn{4}{|c|}{ System perception and evaluation } \\
\hline HQS $(\alpha=.91)$ & $3.48(.88)$ & $2.25(.27)$ & $\mathrm{U}=5.5 *$ \\
\hline $\mathrm{PQ}(\alpha=.72)^{1}$ & $6.03(.89)$ & $5.83(.65)$ & $\mathrm{U}=17$ \\
\hline APPEAL $(\alpha=.86)$ & $4.76(.33)$ & $3.57(.79)$ & $\mathrm{U}=4.5^{*}$ \\
\hline \multicolumn{4}{|c|}{ Task persistence and performance } \\
\hline Number of breaks & $0.00(.00)$ & $1.14(1.22)$ & $\mathbf{U}=9 *$ \\
\hline $\begin{array}{l}\text { Numbers of } \\
\text { addresses }\end{array}$ & $36(5)$ & $39(4)$ & $\mathrm{U}=14$ \\
\hline Duration (minutes) & $67(12)$ & $63(13)$ & $\mathrm{U}=16$ \\
\hline
\end{tabular}

Notes: $\left.{ }^{*}\right) \mathrm{p}<.05 ;{ }^{1}$ ) two items were discarded 
As expected, there was a significant difference in HQS, with a higher stimulation if the pattern was apparent. PQ, however, did not differ between conditions. In general, HQS and PQ did not correlate, Spearman's $\rho(13)=.22$, ns. In other words, PQ and HQS were perceived as sufficiently different aspects and the pattern took effect only on the hedonic, not on the pragmatic quality perceptions.

Further, a significant difference in APPEAL emerged, pointing at the additional value derived from the pattern. Indeed, a stepwise linear regression with PQ and HQS as predictors and APPEAL as criterion showed HQS to be the single best predictor ( $\beta$ $=.82, \mathrm{p}<.01,63 \%$ explained variance, corrected $)$.

Concerning task persistence, a significant difference for number of breaks emerged. With the pattern, participants took no voluntary breaks, whereas without the pattern participants took one break on average. In addition, stimulation was significantly correlated with number of breaks, $\rho(13)=-.62, p<.05$, with less breaks the more the system was perceived as stimulating. No such correlation emerged for PQ, $\rho(13)=.20$, ns. As expected, the remaining task performance measures showed no significant differences.

\section{Conclusion}

All in all, the present study supports the notion that TSD has a positive effect on task persistence and, thus, a motivational effect. Although admittedly preliminary, this does at least not disconfirm our general claim, namely that motivation can be built into software used at the workplace. Obviously, patterns must be used for more complex, real-life applications, and their effects must be studied in the field, too. Nevertheless, we understand the present study as a first step to explore the notion of patterns to engineer motivation and the question of whether they actually work or not.

Acknowledgments. This work is supported by the German Federal Ministry of Education and Research (BMBF) within the project FUN (Grant: 01 IS E06 A). For more information see the project website http://www.fun-of-use.de

\section{References}

1. Constantine, L., Lockwood, L.: Software for Use: A Practical Guide to the Essential Models and Methods of Usage-Centered Design. Addison-Wesley, Reading (1999)

2. Gamma, E., Helm, R., Johnson, R., Vlissides, J.: Design Patterns - Elements of Reusable Object Oriented Software. Addison-Wesley, Reading (1995)

3. Dearden, A., Finlay, J.: Pattern Languages in HCI: A Critical Review. HCI 21, 49-102 (2006)

4. Tidwell, J.: Common ground: A Pattern Language for Human-Computer Interface Design

5. Niebuhr, S., Kohler, K., Graf, C.: Engaging Patterns: Challenges and Means shown at an Example, Engineering Interactive Systems, Salamanca, Spain (2007)

6. Latham, G.P., Lee, T.W.: Goal setting. In: Locke, E.A (ed): Generalizing from laboratory to field settings. Lexington Books, pp. 101-117 (1986)

7. Hassenzahl, M.: The interplay of beauty, goodness and usability in interactive products. Human Computer Interaction 19, 319-349 (2004) 